EXPANDED ABSTRACT

\title{
Tube formation in developing mouse submandibular gland
}

\author{
Yohki Hieda \\ Department of Biological Science, Graduate School of Science, Osaka University and Department of \\ Biology, Osaka Dental University, Osaka, Japan
}

Keywords : submandibular gland, lumen, cell polarity, tight junction, sonic hedgehog

J. Med. Invest. 56 Suppl. : 239-240, December, 2009

Many animal organs are composed of epithelial tubes with a central lumen. In several tubular organs such as the mammary gland, hair follicle, and salivary glands of mammals, the epithelial tissues arise as a solid mass of cells at early developmental stages and later form lumens in the cell masses. This process is closely associated with cell polarization. Cells before lumen formation are unpolarized in that they lack tight junctions (TJs) and the defined plasma membrane domains while cells lining lumens are polarized to have TJs, which separate the plasma membrane into apical or luminal, and basolateral domains. However, the molecular mechanisms of lumen formation in developing organs are poorly understood.

In the mouse submandibular gland (SMG), lumen formation in a solid mass of unpolarized cells begins at embryonic day (E) 14, leading to the establishment of the complete tubular structure lined by polarized cells by E17. The SMG of Sonic hedgehog (Shh) null mice fails to progress to stages beyond around E14, in which the epithelium remains as cell masses (1). On the other hand, as we have shown previously, a treatment with exogenous Shh-N peptide promotes lumen formation of SMG explants (2). As seen in the control glands, lumens in the Shh$\mathrm{N}$-treated glands were delineated by tight junction proteins such as claudins, occludin, and ZO-1. Moreover, aquaporin- 5 was localized at the luminal side

Received for publication October 16, 2009 ; accepted October 23, 2009.

Address correspondence and reprint requests to Yohki Hieda, Department of Biology, Osaka Dental University, 8-1 KuzuhaHanazono, Hirakata, Osaka 573-1121, Japan and Fax : +072-8643163 . of cells while laminin was deposited at the basal side. Thus, Shh-N-induced promotion of lumen formation in the developing SMG is accompanied by the acquisition of polarized nature in cells including the establishment of TJs and defined plasma membrane domains.

Furthermore, our recent study demonstrates involvement of the TJ assembly in lumen formation in the developing SMG. The structure and function of TJs depend on the claudin family of transmembrane proteins, which consist of 24 members in mammals. We previously showed in the developing mouse SMG that claudin- 3 to -8 were expressed along the lumens appearing in the epithelium (3). Notably, it has been reported that the fragment from $\mathrm{COOH}$ terminal half of Clostridium perfringens enterotoxin (C-CPE) binds to an extracellular domain of certain members of the claudin family, including claudin$3,-4,-6,-7,-8$, and to remove claudins from TJs to disorganize the junction structure in cultured cells. When the developing mouse SMG was cultured in the presence of C-CPE, none of the claudin members examined was properly expressed and $\mathrm{TJ}$ formation was inhibited. Furthermore, compared to the control SMG explants, C-CPE-treated explants had remarkably narrow lumens, with apical surface separation of apposing cells being interfered. In addition, the water channel aquaporin- 5 in the treated glands did not localize at the apical plasma membrane but was distributed diffusely on the entire cell surface although NKCC1 localized correctly at the basolateral surface in the absence or presence of $\mathrm{C}$ CPE. Claudins may play a role in lumen expansion by coordinating TJ assembly with biogenesis of the functional apical membrane. 
TJ assembly and apical membrane biogenesis regulated by Shh signaling are suggested to play important roles in lumen formation of the developing mouse SMG. It remains unknown how apical domain of the plasma membrane is established during the formation of initial lumens partly because plasma membrane proteins that marks the initial lumens have not been identified. The identification of such marker proteins will help to understand the mechanism of lumen formation in developing organs.

\section{REFERENCES}

1. Jaskoll T, Leo T, Witcher D, Ormestad M,
Astorga J, Bringas P Jr, Carlsson P, Melnick $\mathrm{M}$ : Sonic hedgehog signaling plays an essential role during embryonic salivary gland epithelial branching morphogenesis. Dev Dyn 229 : 722-732, 2004

2. Hashizume A, Hieda Y : Hedgehog peptide promotes cell polarization and lumen formation in developing mouse submandibular gland. Biochem Biophy Res Commun 339:996-1000, 2006

3. Hashizume A, Ueno T, Furuse M, Tsukita S, Hieda Y : Expression patterns of claudin family of tight junction membrane proteins in developing mouse submandibular gland. Dev Dyn 231 : 425-431, 2004 